\title{
The representation of color and form in long-term memory
}

\author{
AURA HANNA \\ Virginia Commonwealth University, Richmond, Virginia \\ and \\ ROGER REMINGTON \\ NASA-Ames Research Center, Moffett Field, Califormia
}

\begin{abstract}
Color and form are elementary stimulus encoding dimensions that have effects on the representation of visual stimuli at early processing stages. Little is known, however, about their effects on visual long-term memory. In three experiments we investigated whether color is part of the memory representation, whether color and form are bound in the memory representation, and the effect of color context on memory performance. Experimental results suggest that color is part of the memory representation and that color and form can be represented separately in memory and accessed independently. We suggest that the binding of color and form is a deliberate strategic act that requires focal attention, not a natural consequence of processing visual stimuli. We compare our results with the predictions of two computational memory models regarding feature binding. The effect of color context was not straightforward; however, results are consistent with the encoding specificity principle.
\end{abstract}

For most people, color is an integral property of the visual experience. Natural scenes include items whose colors not only enrich our sensory experience but also play an important role in our organization of the visual world. Imagine, for example, a group playing on the lawn of a yellow house. The group might include a man wearing a blue shirt playing with a child wearing green overalls and a dog with an orange ball. Here the varied colors of objects help segregate the world into meaningful objects. This is in contrast to camouflage, where color and color patterns obscure the boundaries between objects. The widespread success of camouflage in nature attests to the importance of color in scene segmentation and hence object identification. Animals have successfully exploited this violation of natural object-color boundaries to confound predators.

In the following experiments, we seek the answers to three questions. First, we are interested in determining whether color is part of the long-term memory representation for an object. Second, we are interested in determining whether the color context in which an item is encoded is important for its later recognition. Third, we are

This research was supported by a National Research Council Postdoctoral Fellowship tenured at the NASA-Ames Research Center and a Virginia Commonwealth University Faculty Grant-in-Aid, both to A. H. The authors thank J. Hartzell for the use of laboratory space at NASA and D. Delgado for assistance with portions of the programming. The authors also thank J. Davidoff, A. Glenberg, G. Loftus, J. Metcalfe, and T. Sanoki for their comments on earlier versions of this manuscript. Correspondence should be addressed to A. Hanna, Psychology Department, Virginia Commonwealth University, Richmond, VA 23284 (e-mail: ahanna@vcu.edu). interested in how color and form are represented in longterm memory. Does access to objects automatically mean access to their color, or is there separate, independent, access to each?

The answers to these questions are of interest for several reasons. Quite a lot has become known recently about how color and form are processed at early stages. At the perceptual stage, color and form are processed in both separate and common areas of the visual cortex (Livingstone \& Hubel, 1988; Zeki et al., 1991). At the attentional stage, when excessive demands are placed on the system, features within a scene, such as color and form, can be bound inappropriately, producing illusory conjunctions (Treisman \& Schmidt, 1982). There is little research that addresses the issue of the binding of color and form in memory directly. There is a similar paucity of research on contextual effects in visual memory. Contextual effects have been well studied in verbal memory, usually as tests of the encoding specificity hypothesis (Tulving \& Thomson, 1973), but little research has been done on contextual effects in visual memory. The question of how color and form are represented in memory - separately or bound together-is of interest because the answer determines the way that visual object information is stored and can be retrieved. It will thus place limits on acceptable models of memory.

\section{Representation of Color}

If color is part of the stored memory representation of an object, it will provide an additional attribute to assist in matching the retrieval cue to the internal representation at retrieval. Thus, when color information is preserved at study and test, one would expect accuracy for 
colored objects to be better than accuracy for black-andwhite objects. Research results confirm this: Recognition is more accurate for colored than for black-andwhite stimuli (Tanaka \& Bunosky, 1993; Wurm, Legge, Isenberg, \& Luebker, 1993). Recognition speed is more difficult to predict. If the matching process is parallel, one might expect recognition speed to be faster for colored objects; if it is serial, recognition speed should slow as a function of the number of attributes to be matched. It turns out, however, that the critical variable is not the type of processing but whether color is diagnostic for the object. The color yellow would be considered diagnostic for a banana whereas the color red would not be considered diagnostic for a hammer. Lack of color is shown to impair recognition speed for objects for which color is diagnostic (Tanaka \& Bunosky, 1993). Thus, if color is part of the learned identity of an object, its presence decreases recognition time.

The effects of color on object identification and classification speed have received more attention that its effects on recognition speed and accuracy. In both classification and identification tasks, advantages for color effects are observed most consistently for well-learned items. For example, in object identification tasks, color decreases reaction time only for natural objects, not for geometric shapes (Davidoff \& Ostergaard, 1988; Ostergaard \& Davidoff, 1985; Price \& Humphreys, 1989). Similarly, subjects are faster to respond to high color-diagnostic items, such as a yellow banana, than to low color-diagnostic items, such as a red hammer (Tanaka \& Bunosky, 1993). Likewise, in classification tasks, correct color facilitates classification speed whereas lack of color and incongruous color inhibit classification speed (Price \& Humphreys, 1989). Results of classification tasks indicate that stimuli need not be natural objects, but they need to be well learned. Murphy (1991) found parallel results using artificial categories: Color could facilitate or inhibit classification at the basic level (cf. Rosch, 1978), depending on whether the color was congruent or incongruent with the learned category. Although the categories were artificial, the colors of the category items were learned.

Color also affects performance on focused attention and integration tasks. Wickens and Andre (1990), for example, compared performance with colored and monochrome aircraft displays. Results showed a speed-accuracy tradeoff for the focused attention task: Accuracy was improved by color at the expense of slowed reaction time. In contrast, for the integration task, color did not improve accuracy; it did slow reaction time.

Color effects have not been apparent in experiments where the stimulus is shown for a relatively brief duration. For example, Biederman and Ju (1988) found that color provided no advantage for the speed of identification of common objects, even when it was diagnostic for the object. Stimuli in Biederman and Ju's experiments were presented for durations of $100 \mathrm{msec}$ or less and were masked, thus constricting processing time to one eye fixation. As with the Wickens and Andre (1990) study, these results suggest that the color representation may be ac- cessed later in processing than other attributes of the stimulus, such as its form.

In general, color confers an advantage either when it is strongly associated with an object's identity or when sufficient processing time is allowed that the object enjoys conceptual, not just perceptual, processing (cf. Hanna \& Loftus, 1993).

\section{Encoding Specificity Principle}

Other evidence suggests that color should aid memory performance only when items are identically colored at study and test. According to the encoding specificity principle (Tulving \& Thomson, 1973), an item to be learned is encoded into a memory representation that includes any extra information about it that was present during encoding. The result is that cues that were part of the original encoded context provide better retrieval cues for recall than cues that were not part of the original context. Similarly, in recognition tests, the more similar the context is at retrieval to that which was present at encoding, the better will be recognition memory performance. Tulving and Thomson stated, "What is stored is determined by what is perceived and how it is encoded, and what is stored determines what retrieval cues are effective in providing access to what is stored" (p. 353). A cue that was not part of the context of the item at encoding will be an ineffective retrieval cue, even if it is a strong associate of the item in semantic memory.

Many experiments in the verbal learning literature have supported the encoding specificity hypothesis. Various contextual effects have been tested, such as by including or omitting studied contextual words at test (Thomson, 1972), manipulating sex of the speaker (Geiselman \& Glenny, 1977), and manipulating congruity of sentence frames (Baker \& Santa, 1977; Morris, 1978). Color context has been tested in a verbal memory paradigm: Murnane and Phelps (1993) manipulated background and foreground color for presentation of word pairs and found that memory performance decreased with changes in color context.

Although the encoding specificity hypothesis has been tested and confirmed repeatedly for verbal stimuli, it has not been tested for visual stimuli.

\section{Conjunction of Color and Form}

Experiments examining whether color and form are bound in the representation generally address early stages of cognitive processing such as object identification and classification and measure reaction time. Results are compared between stimuli where color and form are conjoined and separated. If the conjunction of color and form at test aids performance, the hypothesis that color and form are bound in the representation is supported. There is not much research examining the question, and the results are mixed, with attention tasks leading to conclusions that are different from those generated by object recognition tasks.

The evidence for binding of color and form during encoding comes from studies of attention. According to 
Treisman's feature integration theory (Treisman \& Gelade, 1980), focal attention should result in an integrated object representation. However, when attention is overloaded or distracted, features can be combined inappropriately, producing illusory conjunctions. Treisman and Schmidt (1982) illustrated that when people were instructed to attend to aspects of the display other than the targets, they reported illusory conjunctions of color and form. For example, a person who saw a red $X$ and a green $O$ might report having seen a red $O$. The binding of color and form was also investigated by Stefurak and Boynton (1986) using a short-term recognition memory task. These experimenters used colored animal shapes and varied the study/test, color-form conjunction. They found that when verbal encoding was prevented by an arithmetic task, subjects could not discriminate new color-form conjunctions from old ones.

The question of color-form binding was examined by Price and Humphreys (1989) in an object recognition paradigm using line drawings of structurally similar objects. In one condition, the background color in the display was the correct color for the target object, whereas in the other, the background color was incongruent for the target object. Their reasoning was that, because both color and form are available in the stimulus display, if color and form are represented separately, performance in the target-color background condition should be superior to that in the incongruent-color background. In contrast, if color and form are bound, there should be no advantage to having the target object's color in the background. Results for both reaction times and error data showed no significant performance difference. Price and Humphreys concluded that the representation of color and form is bound and that color affects object recognition only when it is on the object surface. Although Price and Humphreys did not test their assertion directly - that is, there was no condition where the color was on the object surface, Wilton did. Using a technique similar to that of Price and Humphreys, Wilton (1989) compared objects with the color on the object surface or in the background. He showed that both intentional and incidental recall for color was better when color and form were conjoined than when separated.

Results are inconclusive: Treisman and Schmidt's (1982) data and Stefurak and Boynton's (1986) results suggest that color and form are represented separately; Price and Humphreys' (1989) and Wilton's (1989) data suggest that they are bound together.

Current computational memory models, such as CHARM (Metcalfe, Cottrell, \& Mencl, 1992; MetcalfeEich, 1982) and TODAM (Murdock, 1982), make predictions about the binding of features. These models represent stimuli by vectors or matrices composed of elementary features. In both TODAM and CHARM, there is a single composite memory trace. In CHARM, each item is convolved with itself at storage, a process called autoconvolution, before being added into the composite trace. This action ensures binding of features within an item. Thus CHARM would predict that color and form would be bound. In TODAM, by contrast, there is no process analogous to autoconvolution; hence features within an item are not bound. Thus TODAM predicts that color and form will be separate and not bound.

We report three recognition memory experiments that investigate the representation of color and form in memory. Experiment 1 investigates whether color is part of the representation and tests the encoding specificity hypothesis for visual stimuli. Experiments $2 \mathrm{~A}-2 \mathrm{~B}$ are designed to examine the conjunction of color and form in the representation. Because some evidence suggests that it is important to distinguish between verbal and visual coding of visual stimuli (Allen, 1984; Stefurak \& Boynton, 1986), we chose geometric shapes as stimuli to encourage subjects to encode visually rather than verbally. Circles and rectangles were used in the first experiment, and randomly generated quadrilaterals were used in the latter two experiments.

We assume that recognition performance is a function of the match between the stored representation and the test stimulus. We generate sets of predictions for combinations of study/test conditions.

\section{EXPERIMENT 1 Representation of Color}

Our first question was a basic one: Does the presence of color at study and test aid memory performance in a visual memory task? If so, it would imply that color information is represented in long-term memory. To answer the question, we compared performance in conditions in which the forms within a stimulus were identically colored at study and test (color/color) to conditions in which the forms were white with black outlines (bw/bw). Both conditions had congruent study and test items and differed only in the presence or absence of color. If color aids performance, we reasoned, the color condition should lead to better recognition accuracy than the monochrome condition.

The second question we addressed was whether the encoding specificity principle would be observed for a purely visual memory task. To address the encoding specificity hypothesis, we compared congruent study/test conditions (color/color and bw/bw) with incongruent study/test conditions (color/bw and bw/color). If the encoding specifity principle holds for visual memory, then recognition accuracy should be better in the congruent conditions than in the incongruent conditions.

From the above considerations, we generated a set of predictions for combinations of study/test conditions. Four conditions are created by fully crossing the presence or absence of color with study and test. The resulting predictions are as follows:

color $/$ color $>\mathrm{bw} / \mathrm{bw}>$ color $/ \mathrm{bw}=\mathrm{bw} /$ color . 


\section{Method}

\section{Subjects}

Forty-eight subjects were run individually in Experiment 1 . Subjects were San Jose State University students who participated for course credit, NASA staff who volunteered, and individuals from the surrounding community who were paid. All reported normal color vision.

\section{Materials}

Each stimulus was composed of six geometric shapes: six circles, six horizontal rectangles, or six vertical rectangles. All shapes had black outlines. Half the stimuli contained only colored shapes; half contained only white. For the colored stimuli, six colored shapes were in each stimulus: dark blue, yellow, red, brown, green, and purple. White shapes were presented on a white background; colored shapes were presented on a background for which the top half was sky blue and the bottom half green (a different green from that used for the green shapes). The coordinates for each of the six shapes in a stimulus were randomly generated with the restrictions that no shape could exceed one quarter of the screen size and no shape could be totally occluded. Generally, the six geometric shapes took a large portion of the screen. Half the stimuli were assigned as targets; half as distractors. Targets and distractors were made alternately. Each stimulus was made in both a colored and a white version.

\section{Apparatus}

Stimuli were displayed on a 16 -in. NEC Multisync 4D computer screen. Stimuli subtended a visual angle that ranged from $19^{\circ}$ to $20^{\circ}$ horizontal and from $13^{\circ}$ to $15^{\circ}$ vertical. Subjects made all responses on individual two-key response switches. The display and response collection equipment were controlled by an IBM 386compatible computer system.

\section{Design and Procedures}

Study procedure. A trial consisted of 12 sequentially presented stimuli. Each stimulus was presented for $2 \mathrm{sec}$ and followed by a 1 -sec interstimulus interval (ISI). Four experimental conditions were determined by the colors of the stimuli at study and test. In the four conditions, stimuli were black-and-white or colored at study and congruent or incongruent with the study color at test. The four resulting study/test conditions were color $/ \mathrm{color}(\mathrm{col} / \mathrm{col})$, black-and-white/black-and-white (bw/bw), black-and-white/color (bw/col), and color/black-and-white (col/bw). For example, in the bw/col condition, the 12 stimuli in the study trial were white with black outlines; the 24 stimuli in the test were composed of the 12 stimuli seen at study but in their colored versions, randomly mixed with 12 colored distractors. At study, color conditions were presented in blocks; there were three consecutive trials of each of the four color conditions. Within a three-trial color set, each of the three shape conditions (horizontal rectangles, vertical rectangles, circles) was represented in one trial. The experiment was thus composed of 12 study trials and 12 corresponding tests.

Test procedure. After each trial, a recognition test was given that included the 12 study stimuli from the preceding trial along with 12 distractors. In the incongruent conditions, the studied stimuli were in opposite color (colored, if studied in black-andwhite, or black-and-white if studied in color), but each studied stimulus contained the same forms in the same positions. Subjects responded by pressing a key on their response switch. Each test stimulus remained on the screen until the subject responded. Following response, there was a $\mathrm{l}-\mathrm{sec}$ interval prior to presentation of the next test stimulus.

Instructions to subjects. Subjects were informed about the four conditions and about the timing and sequence of upcoming events. To familiarize subjects with the conditions, there was a brief practice trial composed of four study and eight test items prior to each three-trial condition block. For each test stimulus, subjects were asked to respond "yes" or "no" corresponding to whether they thought they had or had not seen the stimulus in the preceding study phase. They were to base their decisions on whether they had seen a stimulus containing the studied geometric shapes, regardless of color. Subjects were instructed to take their time and to try to remember all the stimuli that they had seen.

Design. The design was within subject. The order of the four color conditions was counterbalanced so that each color condition was in each presentation order equally often. For example, for Groups $1-3$, the color condition order was $1-2-3-4$; thus the first three-trial condition block would show Condition 1; the next block, Condition 2; for Groups 4-6, the color condition order was 2-3$4-1$. Within a trial, the 12 stimuli were counterbalanced so that they were presented in three orders: for one third of the groups, the start stimulus was $\# 1$; for one third, it was $\# 5$; and for one third, it was \#9. For example, for Groups 3, 6, 9, and 12, the stimulus order was: 9-10-11-12-1-2-3-4-5-6-7-8. Because we made both black-andwhite and colored versions of each stimulus, a start stimulus contained the same shapes regardless of whether it was colored or blackand-white. Order of the shape condition was constant within the color conditions: horizontal rectangles, vertical rectangles, circles.

\section{Results and Discussion}

A planned comparison using the procedure outlined by Loftus and Loftus (1988) for within-subject designs showed that the data fit the expected pattern of results well $\left[F^{\prime}(1,141)=11.20, p<.01\right]$, accounting for $83 \%$ of the between-condition variance. Weights used in the planned comparison were $3.0,0.0,-1.5$, and -1.5 to fit the predicted pattern of color/color $>\mathrm{bw} / \mathrm{bw}>$ color $/ \mathrm{bw}=$ bw/color. The residual was not significant $\left[F^{\prime}(2,141)=\right.$ $1.15]$.

Means were corrected for guessing so that chance was zero. ${ }^{1}$ These corrected means are presented in Table 1 along with the false alarm rates and uncorrected means. Statistical analyses and textual discussion refer to the corrected means. The standard error of the mean was $0.017 .^{2}$

The answer to our first question was a clear affirmative: Color at study and test (color/color) produced better recognition performance than a congruent study/test condition without color (bw/bw). This outcome supports the hypothesis that color is part of the long-term memory representation. We interpret the advantage of color/ color over bw/bw to mean that, at least for many stimuli, color information was encoded from the visual displays.

The answer to our second question is less clear. Consistent with the encoding specificity hypothesis, perfor-

Table 1

Means and Corrected Means, Experiment 1

\begin{tabular}{lccc}
\hline Condition & Corrected $p$ & False Alarm Rate & Uncorrected $p$ \\
\hline Color/Color & .55 & .21 & .66 \\
BW/BW & .51 & .23 & .63 \\
Color $/ \mathrm{BW}$ & .50 & .29 & .63 \\
BW/Color & .46 & .28 & .60 \\
\hline
\end{tabular}

Note-Color/Color, conditions in which the forms within a stimulus were identically colored at study and test; $\mathrm{BW} / \mathrm{BW}$, conditions in which the forms were white with black outlines at study and test Color/BW, forms were colored at study, white with black outlines at test. BW/Color, forms were white with black outlines at study, colored at test. 
mance was significantly better for conditions that werecongruent at encoding and retrieval than it was for incongruent conditions $[F(1,47)=6.76, p<.05]$. Subjects did better in the color/color and bw/bw conditions than in the color/bw and bw/color conditions. Nevertheless, it is also clear that encoding specificity was not the only factor underlying recognition accuracy. The advantage of color/color over bw/bw cannot be attributed to encoding specificity; nor can the nearly identical accuracy for the $\mathrm{bw} / \mathrm{bw}$ and color/bw conditions. Likewise, encoding specificity provides no basis for the marginally significant advantage of the color/bw condition over the bw/color condition $[F(1,47)=3.66, p<.10]$. In these respects, our predictions were not firmly supported.

One empirical observation of Experiment 1 is that the presence of color at study led to improved recognition accuracy for both congruent and incongruent conditions. That advantage of color at study was identical for congruent and incongruent conditions, suggesting that the two factors, color and congruency, have independent effects on recognition accuracy. We infer from this that both the presence of color in long-term memory and encoding specificity were contributing to performance.

To further examine the role of color in visual memory, Experiment 2 compared performance in a congruent color condition (color/color) to performance in a condition in which the same colors were present in a stimulus at study and test but in which colors were reassigned among the shapes (color/reassigned-color- $\mathrm{col} / \mathrm{r}$-col) at test. By varying the conjunction of color at study and test, we can examine whether color identity is bound to the memory representation of individual shapes.

\section{EXPERIMENT 2A Color-Form Conjunction Varied}

Experiment 1 supported our hypothesis that color is part of the representation of an item in long-term memory. Experiment 2 was designed to examine how color and form are represented in memory. Are they represented separately or bound together? If they are bound, recognition will be facilitated when color and form are correctly combined. Alternatively, color and form could provide independent inputs in the recognition process. Accordingly, recognition will be facilitated as long as the correct color is available in the stimulus display, even if it is not conjoined with the form.

To examine this question, Experiment $2 \mathrm{~A}$ included a reassigned-color $(\mathrm{col} / \mathrm{r}-\mathrm{col})$ condition in which the six colors were reassigned at test among the six forms within a stimulus. In this condition, both the colors and the forms were identical at study and test, but the color-form conjunction was different at test than it was at study. If color and form are bound in the memory representation, memory performance for the color/color condition should be better than that for the color/reassigned-color condition. In both conditions, colors and forms were identical at study and test. Only the conjunction varied.
In Experiments 2A-2B, the stimuli were colored and black-and-white quadrilaterals rather than the circles and rectangles used in Experiment 1. Quadrilaterals were chosen because they could be computer generated and, like the triangles and rectangles, they would be difficult to encode verbally.

We tested the null hypothesis that there would be no difference between the col/col and col/r-col conditions. Consistent with the encoding specificity hypothesis, we predicted that performance in the consistent encodingretrieval conditions would be superior to that in the inconsistent encoding-retrieval conditions. We predicted that performance in the color/bw condition would be superior to that in the bw/color condition, given the marginally significant difference between them in Experiment 1 . Thus, we hypothesized that the pattern of results for encoding-retrieval conditions would be as follows:

$$
\mathrm{col} / \mathrm{col}=\mathrm{col} / \mathrm{r}-\mathrm{col}>\mathrm{col} / \mathrm{bw}>\mathrm{bw} / \mathrm{col} \text {. }
$$

\section{Method}

\section{Subjects}

Fifty-two subjects were run in groups of 1 to 5 in Experiment 2A. Subjects were San Jose State University students who reported normal color vision. They participated for course credit.

\section{Materials}

In Experiments 2A-2B, each stimulus was composed of six randomly generated quadrilaterals rather than the rectangles and circles used in Experiment 1. As in Experiment 1, six colors were in each stimulus; in Experiments 2A-2B, the colors were dark blue, yellow, red, cyan, green, and purple. The colors were similar to those used in Experiment 1 except that we replaced brown with cyan. The materials were otherwise the same as those described in Experiment 1.

\begin{abstract}
Apparatus
In Experiments 2A-2B, the experimental room was set up to permit running of up to 5 subjects at one time. As in Experiment 1, stimuli were displayed on a NEC Multisync 4D computer screen, subjects made responses on individual two-key response switches, and the display and response collection equipment were controlled by an IBM 386-compatible computer system. In Experiments $2 \mathrm{~A}-2 \mathrm{~B}$, stimuli subtended a visual angle that ranged from $9^{\circ}$ to $11^{\circ}$ horizontal and from $6^{\circ}$ to $8^{\circ}$ vertical depending on where the subject sat.
\end{abstract}

\section{Design and Procedure}

Study procedure. As in Experiment 1, a trial consisted of 12 sequentially presented stimuli. Each stimulus was presented for $2 \mathrm{sec}$ and followed by a 1 -sec ISI. Four experimental conditions were determined by the colors of the stimuli at study and test. Three of the four color conditions were identical to those in Experiment 1: $\mathrm{col} / \mathrm{col}, \mathrm{bw} / \mathrm{col}$, and $\mathrm{col} / \mathrm{bw}$. In the fourth condition, stimuli were presented in color at study and tested in color, but the conjunction of color and form within a stimulus was varied at test. In this condition- $\mathrm{col} / \mathrm{r}-\mathrm{col}$ - the arrangement of shapes within a stimulus remained identical between study and test; only the color-form conjunction changed. As in Experiment 1, there were three consecutive trials of each of the four color conditions. The experiment was thus composed of 12 study trials and 12 corresponding tests.

Test procedure. The test procedure was identical to that used in Experiment 1.

Instructions to subjects. Subjects were informed about the upcoming four conditions and about the timing and sequence of 
the events that would occur. As in Experiment 1, there was a brief practice trial composed of four study and eight test items prior to each of the four color conditions. For each test stimulus, subjects were asked to respond "yes" or "no" corresponding to whether they thought they had or had not seen the stimulus in the preceding study phase. As in Experiment 1, they were to base their decisions on whether they had seen a stimulus containing the studied shapes, regardless of color. Subjects were instructed to take their time and to try to remember all the stimuli that they had seen.

Design. The design and counterbalancing was the same as that in Experiment 1.

\section{Results and Discussion}

The planned comparison was significant $\left[F^{\prime}(1,153)=\right.$ $9.44, p<.01]$, accounting for $84 \%$ of the betweenconditions variance. Weights used in the planned comparison were $1.0,1.0,0.0$, and -2.0 to fit the predicted pattern of color $/$ color $=$ color $/ \mathrm{r}$-color $>$ color $/ \mathrm{bw}>\mathrm{bw} /$ color. The residual was not significant $\left[F^{\prime}(2,153)=0.92\right]$.

The $\mathrm{col} / \mathrm{col}$ and $\mathrm{col} / \mathrm{r}$-col conditions were not significantly different from each other $[F(1,51)<1.0]$. Corrected means are presented in Table 2 , along with the false alarm rates and uncorrected means. Statistical analyses and textual discussion refer to the corrected means. The standard error of the mean was .018 .

The lack of difference between the col/col and col/r-col conditions is consistent with the hypothesis that color and form are represented separately. If recognition accuracy depends on an identical match between the retrieval cue and the internal stored representation, as per the encoding specificity principle, our results suggest that color identity per se is not part of an object's memory representation. Had color identity and form identity been bound in memory, performance in the reassigned-color condition would have been poorer than performance in the congruent color condition.

As in Experiment 1, mean performance was better for the $\mathrm{col} / \mathrm{bw}$ condition than for the bw/col condition. Although the difference was not reliable in Experiment 1 , in Experiment 2 it was statistically significant $[F(1,51)=$ $8.41, p<.01]$. The result would appear to be inconsistent with the encoding specificity principle. However, this need not be the case. Our procedure could have led to selective encoding of form in the col/bw condition. In all conditions, the subject was aware of the upcoming test condition. Thus, in the col/bw condition, the subject could encode for form and ignore color, knowing that color would be absent from the test stimulus. At test, the

Table 2

Means and Corrected Means, Experiment 2A

\begin{tabular}{lccc}
\hline Condition & Corrected $p$ & False Alarm Rate & Uncorrected $p$ \\
\hline Color/Color & .49 & .28 & .65 \\
Color/R-Col & .51 & .29 & .66 \\
Color/BW & .50 & .32 & .65 \\
BW/Color & .43 & .31 & .61 \\
\hline
\end{tabular}

Note- - Color/Color, conditions in which the forms within a stimulus were identically colored at study and test. Color/R-Col, conditions in which the same colors were present in a stimulus at study and test but in which colors were reassigned among the shapes at test. Color/BW, forms were colored at study, white with black outlines at test. BW/Color, forms were white with black outlines at study, colored at test. stored representation and the retrieval cue would match. In the bw/col condition, in contrast, the subject could not encode for color; thus there was a mismatch between the stored representation and the retrieval cue.

\section{EXPERIMENT 2B Replication}

In Experiment 2A, recognition memory performance was not dependent on whether color and form were conjoined at test. Performance was equally good whether the color-form conjunction at test was changed from what it was at study or stayed the same. Because we were attempting to accept a null hypothesis of no difference between conditions, it seemed appropriate to replicate the effect. Thus Experiment 2B was a replication of the $\mathrm{col} / \mathrm{col}$ and $\mathrm{col} / \mathrm{r}-\mathrm{col}$ conditions of Experiment $2 \mathrm{~A}$.

\section{Method}

\section{Subjects}

Twenty-six subjects were run in groups of 1 to 5 in Experiment 2B. Subjects were San Jose State University and D'Anza College students who participated for course credit. They reported normal color vision.

\section{Materials}

For the experimental trials, stimuli were the same as those used in Experiment $2 \mathrm{~A}$ for the $\mathrm{col} / \mathrm{col}$ and $\mathrm{col} / \mathrm{r}-\mathrm{col}$ conditions. For the practice trials, new stimuli were computer generated.

\section{Design and Procedures}

Study procedure. As in Experiments 1 and 2A, a trial consisted of 12 sequentially displayed stimuli. Each stimulus was presented for $2 \mathrm{sec}$ and followed by a $1-\mathrm{sec}$ ISI. Two conditions were determined by the colors of the stimuli at study and test: $\mathrm{col} / \mathrm{col}$ and $\mathrm{col} / \mathrm{r}-\mathrm{col} \mathrm{con}-$ ditions, as in Experiment 2A. There were six trials of each of the two conditions, presented in two blocks of three consecutive trials.

In Experiment 2B, there were two practice trials, one for each of the two conditions, presented before the experimental trials. Practice trials were composed of 12 study and 24 test items; that is, they were identical in length to the experimental trials. Practice trials were presented in the order $\mathrm{col} / \mathrm{col}, \mathrm{col} / \mathrm{r}$-col.

Test procedure. The test procedure was identical to that used in Experiments 1 and $2 \mathrm{~A}$.

Instructions to subjects. Subjects were informed about the upcoming two conditions and about the timing and sequence of events that would occur. For each test stimulus, subjects were asked to respond "yes" or "no" corresponding to whether they thought they had or had not seen the stimulus in the preceding study phase. The decision was to be based on whether they had seen a stimulus containing the studied shapes in their studied position, even though the colors of particular shapes in a stimulus might have changed. Subjects were instructed to take their time and to try to remember all the stimuli that they had seen.

Design. The order of the two color conditions in the experiment was counterbalanced so that the order reversed after half of the groups. Each condition was presented for three trials. For Groups 1-3, the order was $\mathrm{col} / \mathrm{col}, \mathrm{col} / \mathrm{r}-\mathrm{col}, \mathrm{col} / \mathrm{col}$, and col $/ \mathrm{r}-\mathrm{col}$. For Groups 4-6, it was reversed. In a trial, the 12 stimuli were counterbalanced so that they were presented in three orders, as in Experiments 1 and $2 \mathrm{~A}$.

\section{Results and Discussion}

An analysis of variance indicated no effect of condition $[F(1,25)<1.0]$. Corrected means, along with corre- 
Table 3

Means and Corrected Means, Experiment 2B

\begin{tabular}{lccc}
\hline Condition & Corrected $p$ & False Alarm Rate & Uncorrected $p$ \\
\hline Color $/$ Color & .41 & .32 & .61 \\
Color $/$ R-Col & .43 & .33 & .62 \\
\hline
\end{tabular}

Note - Color/Color, conditions in which the forms within a stimulus were identically colored at study and test; Color/R-Col, conditions in which the same colors were present in a stimulus at study and test but in which colors were reassigned among the shapes at test.

sponding false alarm rates and uncorrected means, are presented in Table 3. The standard error of the mean was .016 .

The results of Experiment $2 \mathrm{~B}$ were consistent with the $\mathrm{col} / \mathrm{col}$ and $\mathrm{col} / \mathrm{r}-\mathrm{col}$ results from Experiment $2 \mathrm{~A}$ and support the hypothesis that color identity and form are represented separately in memory. They suggest that the color of an object is not bound to its form in the longterm memory representation.

\section{GENERAL DISCUSSION}

We have examined the role of color in recognition memory by testing recognition accuracy under conditions in which color is present at both study and test, only at study, only at test, and neither at study nor test. Both experiments found that the presence of color at study led to better recognition accuracy, even when the study and test stimuli were congruent $(\mathrm{col} / \mathrm{col}>\mathrm{bw} / \mathrm{bw})$. This supports the hypothesis that color is encoded and stored in longterm memory. Experiment 1 also showed that, on average, accuracy for congruent study/test conditions $(\mathrm{col} / \mathrm{col}$ and $\mathrm{bw} / \mathrm{bw}$ ) was better than that for incongruent conditions (col/bw and bw/col), providing some support for the encoding specifity hypothesis in visual memory. Experiments $2 \mathrm{~A}-2 \mathrm{~B}$ included a further condition in which the same colors were present at study and test but assigned to different objects within a stimulus. Performance in the $\mathrm{col} / \mathrm{r}-\mathrm{col}$ condition was indistinguishable from that in the $\mathrm{col} / \mathrm{col}$ condition, where the color of the objects was the same at study and test. This suggests that color identity is not bound closely to object identity in memory. We discuss these issues at greater length below.

\section{Representation of Color}

The results of Experiments 1 and 2A-2B support the hypothesis that color is part of the long-term memory representation. Subjects performed better when color cues were present at encoding and test. These results are consistent with those of experiments measuring differences in accuracy between colored and black-and-white stimuli (Tanaka \& Bunosky, 1993; Wurm et al., 1993). They are also consistent with results of object identification and classification tasks showing that color facilitates reaction time for items that have learned colors (Davidoff \& Ostergaard, 1988; Murphy, 1991; Ostergaard \& Davidoff, 1985; Price \& Humphreys, 1989; Tanaka \& Bunosky, 1993).

\section{Encoding Specificity Principle}

Our results provide some evidence that the encoding specificity hypothesis holds for visual memory as well as verbal memory. Recognition accuracy was better when stimuli were congruent at study and test. This result is consistent with the findings from the large number of experiments in the verbal learning literature (Baker \& Santa, 1977; Geiselman \& Glenny, 1977; Morris, 1978; Thomson, 1972) but has heretofore not been reported for visual stimuli. Clearly, as mentioned earlier, encoding specificity does not provide a full account of the data. Indeed, recognition accuracy in the incongruent $\mathrm{col} / \mathrm{bw}$ condition is equal to that of the congruent bw/bw (Experiment 1) and col/col (Experiment 2A) conditions. The bw/col condition was the only incongruent condition showing consistently poorer recognition scores. We have speculated above that this pattern of results may have arisen from selective encoding, not from a violation of the encoding specificity principle. Since subjects knew that the test stimuli in the col/bw condition would not contain color, they could have focused their attention on the form information. However, in the bw/col condition, subjects could not have done anything at study to compensate for the presence of incongruent color at test. Garner has shown that filtering of color is possible though not perfect (Garner, 1983; Gottwald \& Garner, 1972). Focusing attention on form could have aided retrieval in at least one of two ways. First, if the filtering had been perfect (at least for some stimuli), only form information would have been encoded and stored. Thus, the white test stimuli would have been a close match to the stored representation. Second, focusing attention on form information could have selectively enriched the representation of form. Increasing the information about form could have offset the disadvantage of not having retrieval cues that perfectly match the stored items. Thus, there are still ways to reconcile our results within the encoding specificity framework. It would be of interest, though, to explore conditions in which subjects were unaware of the nature of the test stimuli, or were misinformed. Such conditions would provide a stronger test of encoding specificity.

\section{Conjunction of Color and Form}

Results of Experiments 2A-2B are consistent with the hypothesis that color and form are represented separately in memory. The $\mathrm{col} / \mathrm{col}$ and $\mathrm{col} / \mathrm{r}-\mathrm{col}$ conditions showed equivalent memory performance. Clearly, color identity is not bound strongly to object identity in memory. The results of Stefurak and Boynton (1986) support this conclusion. They found that, although subjects could recognize color and form, they were unable to recognize correctly the conjunction of color and form.

Why, then, does color at study and test improve recognition accuracy even when the specific colors assigned to each item differ? One possibility, consistent with the encoding specificity principle, is that the presence or absence of color is stored with the object, but not its spe- 
cific color value. It is not clear how reasonable such a hypothesis is.

A similar account is that color is not bound to specific objects during encoding unless attention is specifically devoted to the binding and a deliberate attempt is made to relate colors to specific objects. Thus, in experiments such as Wilton's (1989), subjects are expected to code both color and form and produce verbal codes that relate the two. In our experiments, however, we did not require subjects to report the binding of color and form. Instead, the color advantage shown in our experiments could have occurred if color and form were both encoded, but not bound together. Given our display times, coupled with the stimuli composed of multiple geometric forms, it is unlikely that deliberate color/form conjunctions would have been made, especially if the conjunction process is serial. Encoding and storing separate color patterns in addition to form patterns enables retrieval by either color or form cues, thus improving the chances of retrieving a previously presented stimulus. Since our task was difficult, the redundancy provided by the extra retrieval cue had a substantial chance of increasing recognition scores. This account suggests that attention is required for binding of features.

Treating the binding of color and form in memory as a deliberate act requiring focal attention helps reconcile our results with existing data. We infer separate representations for color and form from the equivalent performance in our identical-color and reassigned-color conditions. Price and Humphreys (1989) found similarly equivalent identification response times for congruent and incongruent color conditions, but concluded that color and form are bound in the representation. Recall that in their experiments the target object was always white, and the background color at test was either congruent or incongruent with the learned color for the target object. Selective attention to the target at study could have resulted in filtering out color information. Thus, their result poses no problem for our account, since it is not clear that the color information in their experiments was ever coded and stored.

Color is just one stimulus feature. The conjunction of other features has been investigated. For example, recognition memory for facial features has been investigated by Reinitz and his colleagues (Reinitz, Lammers, \& Cochran, 1992; Reinitz, Morrissey, \& Demb, 1994) in experiments manipulating both encoding times and attentional demands. When attention was divided, results were parallel to our own: Subjects were unable to distinguish old target faces from conjunction faces composed of features from two separately studied faces (Reinitz et al., 1994). The latter results again suggest that features are represented independently and can be accessed independently. Binding of features requires focal attention.

Metcalfe, Kersey, and Cottrell (1993) have run a series of experiments on face recognition examining the effects of conjunctions formed from the features of two studied faces and blends formed from photographically superimposed composites of two studied faces. Experi- mental results were similar to those obtained by Reinitz and his colleagues in their full attention experiments: Memory performance was better for previously seen target faces than for conjunctions or superimposed composites from two previously seen faces. Metcalfe and her colleagues fit these results to her CHARM model (Metcalfe, 1982), Hintzman's (1986) MINERVA 2 model, and Murdock's (1982) TODAM model. Because of its autoconvolution process, which provides for binding of features within a stimulus, CHARM can predict these results. In contrast to CHARM, TODAM predicts equivalent performance for previously seen target faces, conjunctions, and composites.

TODAM, which does not provide for feature binding, would predict our results showing no difference in memory performance between the $\mathrm{col} / \mathrm{col}$ and $\mathrm{col} / \mathrm{r}-\mathrm{col}$ conditions and Reinitz, Morrissey, and Demb's (1994) results in the divided-attention conditions. Because CHARM predicts within-item feature binding, it would not predict our results showing equivalent performance for the $\mathrm{col} / \mathrm{col}$ and $\mathrm{col} / \mathrm{r}-\mathrm{col}$ conditions.

\section{CONCLUSIONS}

We have identified conditions under which the representation of color and form appear to be separate. The existing evidence is, in general, consistent with the conclusion that color and form information can be integrated if the task requires it, but that such integration is a deliberate strategic response to a specific set of conditions, not the natural consequence of encoding. Rather, in conditions such as ours, where the binding of color and form is not explicitly tested, the stored representation appears to contain both color and form information that can be accessed separately.

\section{REFERENCES}

Allen, C. (1984). Short-term memory for colors and color names in the absence of vocalization. Perceptual \& Motor Skills, 59, 263266.

BAKER, L., \& SANTA, J. L. (1977). Context, integration, and retrieval. Memory \& Cognition, 5, 308-314.

Biederman, I., \& JU, G. (1988). Surface versus edge-based determinants of visual recognition. Cognitive Psychology, 20, 38-64.

DAvidofF, J., \& OSTERGAARD, A. (1988). The role of color in categorical judgments. Quarterly Journal of Experimental Psychology, 40A, 533-544.

GARNER, W. R. (1983). Asymmetric interactions of stimulus dimensions in perceptual information processing. In T. Tighe \& B. Shepp (Eds.), Perception, cognition, and development: Interactional analyses (pp. 1-38). Hillsdale, NJ: Erlbaum.

Geiselman, R. E., \& Glenny, J. (1977). Effects of imagining speakers' voices on the retention of words presented visually. Memory $\&$ Cognition, 5, 499-504.

GotTWALD, R. L., \& GaRNER, W. R. (1972). Effects of focusing strategy on speeded classification with grouping, filtering, and condensation tasks. Perception \& Psychophysics, 11, $179-182$.

Hanna, A., \& Loftus, G. R. (1993). A model for conceptual processing of naturalistic scenes. Canadian Journal of Experimental Psychology, 47, 548-569.

HiNTZMAN, D. (1986). "Schema abstraction" in a multiple-trace memory model. Psychological Review, 93, $411-428$

Livingstone, M., \& Hubel, D. (1988). Segregation of form, color, 
movement, and depth: Anatomy, physiology, and perception. Science, 240, 740-749.

Loftus, G. R., \& LofTus, E. (1988). Essence of statistics (2nd ed.). New York: Knopf.

LofTUS, G. R., \& MASSON, M. E. (1994). Using confidence intervals in within-subject designs. Psychonomic Bulletin \& Review, 1, 476-490.

MetCalfe, J., Cottrell, G., \& MenCl, W. (1992). Cognitive binding: A computational-modeling analysis of a distinction between implicit and explicit memory. Journal of Cognitive Neuroscience, 4 289-298.

Metcalfe, J., Kersey, M., \& Cottrell, G. (1993, November). Binding in explicit memory. Paper presented at the annual meeting of the Psychonomic Society, Washington, DC.

MetCALfE-EICH, J. (1982). A composite holographic associate recall model. Psychological Review, 89, 627-661.

MORRIs, C. (1978). Acquisition-test interactions between different dimensions of encoding. Memory \& Cognition, 6, 354-363.

MuRDOCK, B. (1982). A theory for the storage and retrieval of item and associative information. Psychological Review, 89, 609-626.

Murnane, K., \& Phelps, M. (1993). A global approach to the effect of changes in environmental context on recognition. Journal of Experimental Psychology: Learning, Memory, \& Cognition, 19, 882894.

MuRPHY, G. L. (1991). Parts in object concepts: Experiments with artificial categories. Memory \& Cognition, 19, 423-438.

OstergaARD, A., \& Davidoff, J. (1985). Some effects of color on naming and recognition of objects. Journal of Experimental Psychology: Learning, Memory, \& Cognition, 11, 579-587.

Price, C., \& HumphreYs, G. (1989). The effects of surface detail on object categorization and naming. Quarterly Journal of Experimental Psychology, 41A, 797-828.

ReINITZ, M., LAMMERS, W., \& COCHRAN, B. (1992). Memory conjunction errors: Miscombination of stored stimulus features can produce illusions of memory. Memory \& Cognition, 20, 1-11

REINITZ, M., MORRISSEY, J., \& DEMB, J. (1994). Role of attention in face encoding. Journal of Experimental Psychology: Learning. Memory \& Cognition, 20, 161-168.

Rosch, E. (1978). Principles of categorization. In E. Rosch \& B. Lloyd (Eds.), Cognition and categorization (pp. 27-48). Hillsdale, NJ: Erlbaum.

StefuRAK, D., \& Boynton, R. (1986). Independence of memory for categorically different colors and shapes. Perception \& Psychophysics, 39, 164-174.

TANAKA, J., \& BUNOSKY, L. (1993, November). Is the object recogni- tion system really colorblind? Poster presented at the annual meeting of the Psychonomic Society, Washington, DC.

ThOMSON, D. (1972). Context effects in recognition memory. Journal of Verbal Learning \& Verbal Behavior, 11, 497-511.

Treisman, A., \& Gelade, G. (1980). A feature integration theory of attention. Cognitive Psychology, 12, 97-136.

Treisman, A., \& Schmidt, H. (1982). lllusory conjunction in the perception of objects. Cognitive Psychology, 14, 107-141.

Tulving, E., \& Thomson, D. (1973). Encoding specificity and retrieval processes in episodic memory. Psychological Review, 80, 352 373.

WiCKENS, C., \& ANDRE, A. (1990). Proximity compatibility and information display: Effects of color, space and objectness on information integration. Human Factors, 32, 61-77.

WILTON, R. (1989). The structure of memory: Evidence concerning the recall of surface and background color of shapes. Quarterly Journal of Experimental Psychology, 41, 579-598.

Wurm, L., LegGe, G., IsenberG, L., \& LuebKer, A. (1993). Color improves object recognition in normal and low vision. Journal of Experimental Psychology: Human Perception \& Performance, 19, 899911.

Zeki, S., Watson, J., Lueck, C., Friston, K., Kennard, C., \& FrackOWIAK, S. (1991). A direct demonstration of functional specialization in human visual cortex. Journal of Neuroscience, 11, 641-649.

\section{NOTES}

1. All reported results were corrected for guessing using the following formula:

$$
p(R n)=[p(H)-p(F A)] /[1-p(F A)],
$$

where $P(R n)$ is the probability of recognition, $P(H)$ is the probability of a hit, and $p(F A)$ is the false-alarm rate.

2. The standard error of the mean in this within-subject design was computed with the following formula:

$$
S E_{\bar{x}}=\sqrt{M S_{S \times C} / n},
$$

where $S E_{\bar{x}}$ is the standard error of the mean, $M S_{S \times C}$ is the mean square, subject $\times$ condition interaction, and $n$ is the number of subjects (Loftus \& Masson, 1994).

(Manuscript received May 31, 1995; revision accepted for publication July 19,1995 . 